\title{
Patient-Reported Outcome and Healing Biomarkers in Patients Treated by Female versus Male Surgeons - A cohort study on Achilles tendon ruptures
}

\author{
J. Flodin', ${ }^{1,3}$ R. Juthberg ${ }^{1,3}$, G. Edman², P. Ackermann ${ }^{1,3}$ \\ 1 Integrative Orthopedic Laboratory, Department of Molecular Medicine and Surgery, \\ Karolinska 1, Stockholm, Sweden \\ 2 Department of Psychiatry, Tiohundra AB, Norrtälje, Sweden \\ 3 Department of Orthopedic Surgery, Karolinska University Hospital, Stockholm, Sweden
}

\section{CORRESPONDING AUTHOR:}

Paul Ackermann

Dept. Orthopedics, A2:07

Karolinska University Hospital SE-171 76 Stockholm, Sweden E-mail: Paul.Ackermann@sll.se Phone: +468517 71734

DOI:

10.32098/mltj.04.2019.07

LEVEL OF EVIDENCE: 3B

\begin{abstract}
SUMMARY
Background. Woman and men practice medicine differently. One recent study showed that patients operated on by female surgeons exhibited better outcome. We hypothesized that the sex of the surgeon would not affect patient outcome, adverse events and the biological processes of healing after surgical repair of Achilles tendon rupture (ATR).

Methods. A total of 111 patients with acute ATR were surgically repaired by the surgeon on duty. A specific surgeon could not be decided by the patient. At two-weeks, levels of healing biomarkers collagen I and III were quantified in microdialysates from the operated tendons. At 12 -months post-surgery, patient-reported outcome was assessed using the validated Achilles tendon Total Rupture Score (ATRS), and adverse events were measured.

Results. Patients treated by female versus male surgeons exhibited higher levels of collagen I $(\mathrm{p}=0.034)$ and reported better ATRS ( $88 \pm 8.2$ vs. $81 \pm 14, \mathrm{p}=0.011)$. Moreover, patients reported less limitations in daily living activity, more strength and less stiffness in the ankle when treated on by female as compared to male surgeons (all $\mathrm{p}<0.05$ ). No significant difference in the incidence of adverse events was observed between male and female surgeons.

Conclusions. The present findings suggest further exploration of the determinating factors including care and communication related to surgeons to improve outcome.
\end{abstract}

KEY WORDS

Achilles tendon; postoperative complications; patient reported outcome measures; rupture; sex of the surgeon

\section{INTRODUCTION}

Patient outcome after surgical interventions is still suboptimal in many areas, such as in Achilles tendon rupture (ATR) repair (15), and patients may suffer from residual symptoms such as pain, weakness, and limited range of motion.

Considering surgically treated patients, there are studies indicating that characteristics of the surgeon, such as training background, age and surgery frequency, affect patient outcomes $(4,7,16)$. However, if the sex of the surgeon is related to patient outcome, has been a controversial issue in the past, as indicated by the limited number of studies on this subject $(18,19,22)$.
From the few studies conducted in this field, it has been shown that female physicians use more patient-centered communication $(2,17)$ and are more likely to follow clinical guidelines (3). One large observational study found that patients treated by female, as compared to male internists, not only exhibited a lower 30-day readmission, but more importantly, a lower 30-day mortality (20). Similarly in surgical fields, recent studies indicate a lower mortality-rate among patients operated on by female surgeons $(18,22)$. One study including a total of 45826 surgeons, found that female surgeons aged 50-59 years exhibited the lowest operative mortality-rates across all examined groups of surgeons (19). Earlier register studies have, however, not 
identified potential explanations for differences in outcome observed between female and male surgeons.

Recently, however, a method to assess the healing progression of ATR was developed by using a microdialysis technique followed by quantification of tissue-repair biomarkers.1 Higher levels of tendon building blocks, normalized collagen type-I (n-PINP) and type-III (n-PIIINP), were associated with improved self-reported outcome twelve months after ATR (1).

Thus, the aim of the present study was to go one step further, not only examining potential discrepancies in patient-reported outcome related to surgeon sex, but to explore whether biological pathways in the underlying healing processes of patients could differ between female and male surgeons. We hypothesized that the sex of the surgeon would not affect healing biomarkers, adverse events and patient-reported outcome after ATR surgical repair.

\section{MATERIALS AND METHODS}

Ethical approval was obtained from the Regional Ethical Review Committees (Dnr: 2013/1791-31/3, 2009/2079$31 / 2$ ) and the original trials was listed in clinicaltrials.gov.

\section{Patients}

Two hundred and fifty-five patients with unilateral acute Achilles tendon rupture (ATR) were retrospectively included from two different randomized control trials (RCTs) at the Karolinska University Hospital, Sweden (figure 1).

All patients were operated on according to a standardized surgical protocol and randomized to three different post-operative treatments. Surgeons on duty performed the surgery and no specific surgeons were selected by the patients. The inclusion criteria were acute unilateral ATR in patients aged between 18 and 75 and the exclusion criteria as earlier published $(5,21)$. Unknown sex of the operating surgeon was included as an additional exclusion criterion in the study. In order to assess the biological factors associated with tendon healing, willingness to perform microdialysis at the two-week follow-up was added as an inclusion criterion in this study. Based on inclusion and exclusion criteria, a total of 111 patients were included in the study with 80 patients operated on by male and 31 patients operated by female surgeon (figure $\mathbf{1}$ ).

\section{Sex of the surgeon and patient demographics}

None of the patient characteristics (sex, age, height, weight, BMI and smoking), time to surgery or the operative time were significantly correlated to the sex of the surgeon or the different adverse events (table I).

\section{The professional experience and age of the surgeon}

The experience of the surgeon was registered as a potential confounding factor. The general experience of the surgeon was defined as being a specialist $(=1)$ or resident $(=0)$ in the orthopedic field. However, male surgeons were more likely to be specialists since the sex of the surgeon (male $=0$, female $=1$ ) was significantly correlated to the general experience $(\mathrm{r}=-.233$, $\mathrm{p}=.013$ ). To verify the results the factor of being a specialist or not was controlled for in the regression analysis.

A total of 32 male surgeons performed 80 ATR surgeries while 31 ATR surgeries were performed by 9 female surgeons. Male surgeons were older than female with a mean age for male of $44 \pm 9$ years and for female surgeons $38 \pm 6$ years $(p=0.009)$. The age of the surgeon was not correlated to any of the patient characteristics, adverse events or outcome variables.

\section{Surgical procedure}

All patients were operated according to the standardized surgical protocol and standardized local anesthetic was used. Briefly, the surgical procedure was initiated by a medial incision over the Achilles tendon, followed by a central incision through the fascia cruris and the paratenon. A modified Kessler suture technique, using two 1-0 polydioxanone (PDS II) sutures (Ethicon, Sommerville, New Jersey, USA) was used to suture the tendon ends together. Thereafter, the paratenon and fascia cruris were sutured separately using 3-0 Vicryl sutures, and skin was closed with 3-0 Ethilon sutures (Ethicon, Sommerville, New Jersey, USA). All patients were prescribed paracetamol $500 \mathrm{mg} /$ codeine $30 \mathrm{mg}$ for post-operative pain. No anti-inflammatory or thromboprophylactic drugs were given to the patients.

\section{Post-operative treatments and rehabilitation}

The patients were randomized to either a plaster cast $(n=38)$ $(5,21)$, an orthosis with an intermittent pneumatic compression (IPC) device beneath $(\mathrm{n}=18)$ (5) or an ankle-mobile orthosis ( $\mathrm{n}=55$ ) (21) (VACO®ped, OPED Gmbh, Germany). The post-operative treatments varied between patients during the first two weeks after surgery. There were no significant correlations between the different post-operative treatments and the sex of the surgeon, the different outcome variables or the adverse events. 


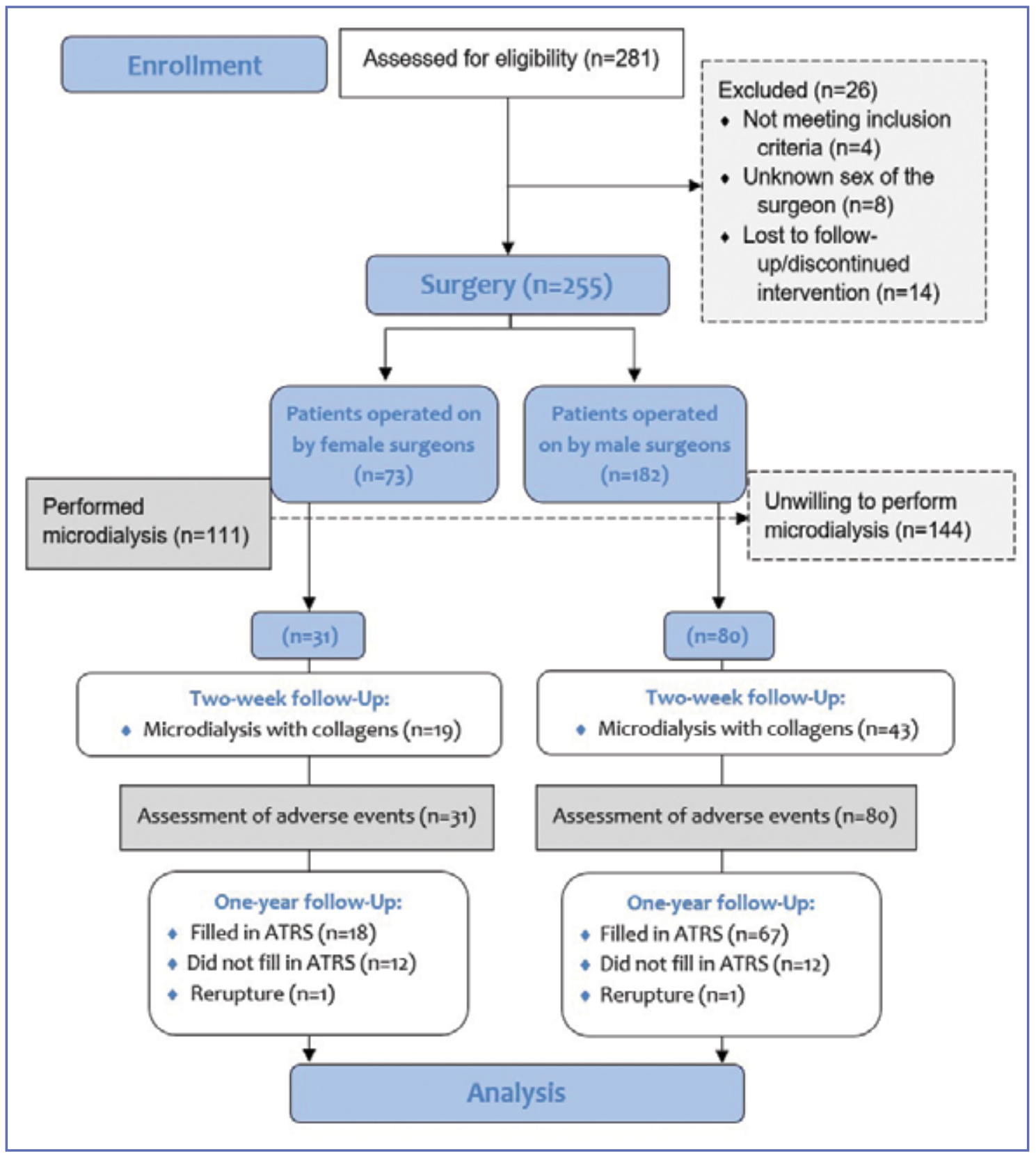

Figure 1. Flowchart describing patients and follow-up. 111 patients were included in the study. Abbreviations: ATRS $=$ Achilles tendon Total Rupture Score.

\section{Microdialysis}

At week 2 post-surgery, microdialysis was performed on all patients as described previously8. Briefly, a small catheter was inserted at the ventral aspect of the paratenon and close to the rupture site, under local anesthesia. Microdialysates were collected at the speed of $1.0 \mu \mathrm{L} / \mathrm{min}$. The samples were collected every $30 \mathrm{~min}$ during a period of $2 \mathrm{~h}$ and immediately stored at $-80^{\circ} \mathrm{C}$ for future analysis. Microdialysis was conducted by one investigator blinded to the study procedure.

\section{Collagen I and III assessment by ELISA}

To assess markers of healing, the $\mathrm{N}$-terminal peptides of procollagen I (PINP) and procollagen III (PIIINP) were 
Table I. Patient characteristics: 111 patients were included in the study.

\begin{tabular}{|c|c|c|c|c|}
\hline \multirow[t]{2}{*}{ Variable } & & \multicolumn{3}{|l|}{ Patients operated on by: } \\
\hline & & Male surgeons $(n=80,72 \%)$ & $\begin{array}{l}\text { Female surgeons }(n=31, \\
28 \%)\end{array}$ & P-value \\
\hline Age, (Years) & $\mathrm{M} \pm \mathrm{SD}$ & $41 \pm 8$ & $39 \pm 6$ & .451 \\
\hline Height, $(\mathrm{cm})$ & $\mathrm{M} \pm \mathrm{SD}$ & $178.5 \pm 8.4$ & $176.6 \pm 8.3$ & .306 \\
\hline Smoker & $\mathrm{n}(\%)$ & $2(3)$ & $2(7)$ & .311 \\
\hline $\begin{array}{l}\text { Time to surgery, } \\
\text { (Hours: Minutes) }\end{array}$ & $\mathrm{M} \pm \mathrm{SD}$ & $80: 17 \pm 43: 50$ & $87: 23 \pm 55: 58$ & .529 \\
\hline Operative time, (Minutes) & $\mathrm{M} \pm \mathrm{SD}$ & $40 \pm 15$ & $37 \pm 15$ & 1.0 \\
\hline Rerupture & $\mathrm{n}(\%)$ & $1(1)$ & $1(3)$ & .482 \\
\hline Infection & $\mathrm{n}(\%)$ & $2(3)$ & $1(3)$ & 1.0 \\
\hline
\end{tabular}

measured in the micro dialysate using a commercially available ELISA kit (USCN Life Science, Inc., Houston, TX, USA). All assays were performed simultaneously, according to the protocol set by the manufacturer. Protein content was measured by using Bradford assay. The normalized procollagen levels were calculated by dividing the concentrations of PINP and PIIINP by the total protein content.

\section{Subjective patient-reported outcome}

The ATRS is a validated and highly reliable questionnaire used to assess patient outcome 12 months after ATR-surgey (14). The ATRS includes 10 specific questions regarding pain, strength, tiredness or stiffness, and physical activities including jumping, running, walking on uneven surface or in stairs and scored from 0 - 10, with $0=$ worst and $10=$ best. The maximum total ATRS-score is 100 , signifying the best possible outcome.

\section{Adverse events}

Adverse events, such as peri-operative surgical complications, DVTs, wound infections and re-ruptures, were recorded throughout the original studies and treated according to hospital protocol. Because of the low frequency of events within each of the above-mentioned variables, these were combined into a single composite variable.
All patients were screened for DVT in the operated leg by unilateral compression duplex ultrasound (Philips CX 50 ultrasound machine, Philips Medical Systems, Andover, MA, USA) at the two- and six-week follow-up. All deep proximal and distal veins, including muscle veins and $\mathrm{v}$. saphena magna were evaluated by two experienced ultrasonographists, who were blinded to the sex of the surgeon. The criteria for DVT diagnosis and the diagnostic procedure have been described earlier $(5,13)$. If the patient had any signs of wound infection, a culture was taken to diagnose a potential infection at the two- and six-week followup. Peri-operative surgical complications, such as if the surgeon needed to call on a fellow surgeon or elongate the incision, were considered as complications and were assessed by journal reviewing.

\section{Statistical analysis}

A power calculation was based upon data from a clinically relevant difference in patient-reported outcome, ATRS, of 7 points (6). We estimated that one group would at least achieve 80 points in ATRS. Thirty-three patients in each group, were required to detect a difference of 7 points in ATRS at one year (two-sided type-I error rate $=5 \%$; power $=80 \%$ ).

The data were analyzed using SPSS version 24 (IBM Corp. Released 2016. IBM SPSS Statistics for Windows, Armonk, NY: IBM Corp.) in cooperation with a statistician. All vari- 
ables were summarized with standard descriptive statistics such as mean, standard deviation and frequency. Student's t-test was applied to detect differences between groups for normal-distributed variables. For non-normal distributed data, Mann-Whitney U-test and Fishers exact test (two-sided) were applied. The significance of correlations was determined by parametric Pearson or non-parametric Spearman correlation coefficient. For variables that were significantly correlated, or close to correlation, multiple linear regressions analyses (stepwise forward method, with an inclusion level of .05 and exclusion level of .10) were applied. This was done in order to investigate the unique relationships between the independent patient variables (sex, age, smoker) and surgeon experience, as well as the relationships between time from injury to surgery and the dependent variable. The significance level in all analyses was set at $\mathrm{p} \leq .05$ (two-tailed).

\section{RESULTS}

\section{Patient-reported outcome}

At the one-year follow-up, patients operated on by female surgeons exhibited significantly better total ATRS compared to patients operated on by male surgeons (figure 2). In the specific ATRS-questions, patients operated on by female surgeons exhibited significantly less limitations in ADL, less stiffness, more strength in the calf/tendon and less limitations on uneven surfaces (all $\mathrm{p}<.05)$. Regression analyses corroborated significant differences for less limitations in

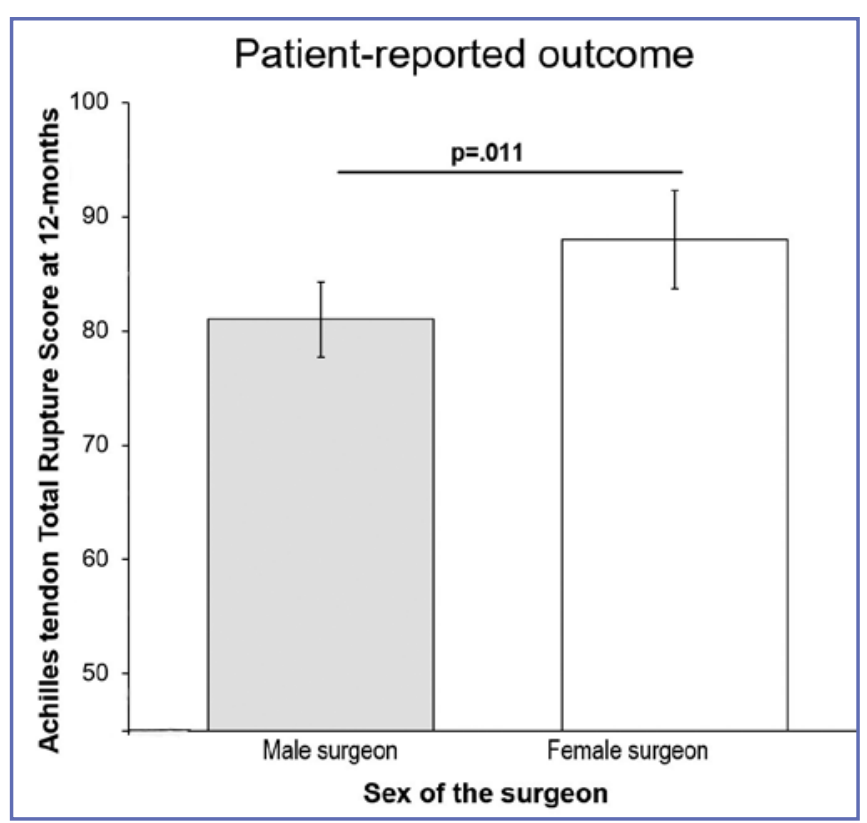

Figure 2. Total ATRS for patients operated on by female $(n=18)$ and male surgeons $(n=66)$ with $95 \% \mathrm{Cl} .84$ patients were included in the analysis.

ADL, less stiffness and more strength in the calf/tendon as measured after controlling for potential confounding variables (patient sex, age, smoking, surgeon experience and the time from injury to surgery) (table II).

Table II. Subjective outcomes. Out of 111 microdialysis-patients, 85 answered the ATRS. Patient characteristics (sex, age, smoker), surgeon sex and surgeon experience were considered as independent variables in the multiple linear regression.

\begin{tabular}{lllll}
\hline Outcome & T-test & & \multicolumn{1}{l}{ Multiple linear regression } \\
\hline & Male surgeon & Female surgeon & p-value ${ }^{\text {a }}$ & \\
\hline Patients reported outcome & & & & \\
\hline ATRS total sum & $81 \pm 14$ & $88 \pm 8.2$ & $\mathbf{. 0 1 1}$ & $\mathrm{F}(1.80)=5.286, \mathrm{p}=.024, \mathrm{R}^{2}=0.062$ \\
\hline Limited strength in calf/tendon/foot? & $6.9 \pm 2.2$ & $8.5 \pm 1.2$ & $\mathbf{. 0 0 5}$ & $\mathrm{F}(1.81)=8.662, \mathrm{p}=.004, \mathrm{R}^{2}=0.097$ \\
\hline Tired in the calf/tendon/foot? & $8.0 \pm 1.8$ & $8.4 \pm 1.9$ & .427 & \\
\hline Stiffness in the calf/tendon/foot? & $7.5 \pm 2.0$ & $8.9 \pm 1.2$ & $\mathbf{. 0 0 7}$ & $\mathrm{F}(1.81)=8.132, \mathrm{p}=.006, \mathrm{R}^{2}=0.091$ \\
\hline Pain in the calf/tendon/foot? & $9.1 \pm 1.4$ & $9.5 \pm 0.9$ & .363 & \\
\hline Limited ADL? & $8.8 \pm 1.6$ & $9.7 \pm 0.6$ & $\mathbf{. 0 1 9}$ & $\mathrm{F}(1.81)=4.962, \mathrm{p}=.029, \mathrm{R}^{2}=0.058$ \\
\hline Limited on uneven surface? & $8.8 \pm 1.6$ & $9.5 \pm 1.0$ & $\mathbf{. 0 4 5}$ & $\mathrm{F}(1.80)=3.294, \mathrm{p}=.073, \mathrm{R}^{2}=0.040$ \\
\hline Limited in stairs/hills? & $8.7 \pm 1.6$ & $9.2 \pm 1.0$ & .419 & \\
\hline Limited when running? & $7.6 \pm 2.2$ & $7.8 \pm 2.4$ & .713 & \\
\hline Limited when jumping? & $6.9 \pm 2.3$ & $7.7 \pm 1.7$ & .121 & \\
\hline Limited in physical work? & $8.7 \pm 1.6$ & $9.0 \pm 1.0$ & .700 & \\
\hline
\end{tabular}

${ }^{a}$ Statistically significant values in bold.

ATRS=Achilles tendon Total Rupture Score; ADL=Activity of Daily Life 


\section{Biomarkers of Tendon callus production}

Tendon healing was monitored by studying levels of collagen I (n-PINP) and III (n-PIIINP) in microdialysate. We observed higher levels of n-PINP in patients operated on by female $(6.6 \pm 4.9)$ compared to male surgeons $(4.7 \pm 3.2)$, which was close to significant $(\mathrm{p}=0.053)$. Regression analyses verified significant differences $(\mathrm{p}=0.034)$ in $\mathrm{n}$-PINP levels after controlling for confounding variables (patient sex, age, smoking, surgeon experience and the time from injury to surgery) (figure 3). Patients operated on by female surgeons exhibited higher levels $(122 \pm 77)$ of n-PIIINP than by male surgeons $(94 \pm 50)$, however the differences were non-significant $(\mathrm{p}=0.158)$.

\section{Adverse events}

There were no significant differences in the rate of adverse events among patients operated on by male versus female surgeons ( $\mathrm{p}=.4$; table $\mathbf{I})$. The incidence of adverse events in the injured leg at the two- and six-week follow-up were $48 \%(15 / 31)(95 \%$ CI, 31\% to 66\%) among patients operated on by female surgeons and 39\% (31/80) $(95 \%$ CI, $28 \%$ to $49 \%$ ) among patients operated on by male surgeons (table I).

\section{DISCUSSION}

This study is the first to investigate how patient outcome and levels of biomarkers related to the healing-process in Achilles tendons varied between patients operated on by male and by female surgeons. The study demonstrates that the patients operated on by female surgeons exhibited significantly better patient-reported outcome, i.e. less symptoms, according to total ATRS one year after ATR-surgery compared to patients operated on by male surgeons. In this study patients operated on by female surgeons also exhibited significantly higher levels of biomarkers that possibly could be related to the sex of the surgeon. The risk of sustaining adverse events was similar between patients operated on by female and male surgeons.

This study established that patients operated on by the female surgeons exhibited significantly better total ATRS, a mean of 88 points, while patients operated on by the male surgeons exhibited 81 points. A difference of 7 points in the total ATRS has in an earlier study been considered as a clinically relevant difference (6).

The difference in the total ATRS among patients operated on by female vs male surgeons can partly be explained by the significantly better ATRS-subscores in three of the ten individual ATRS-questions. Specifically, patients operated

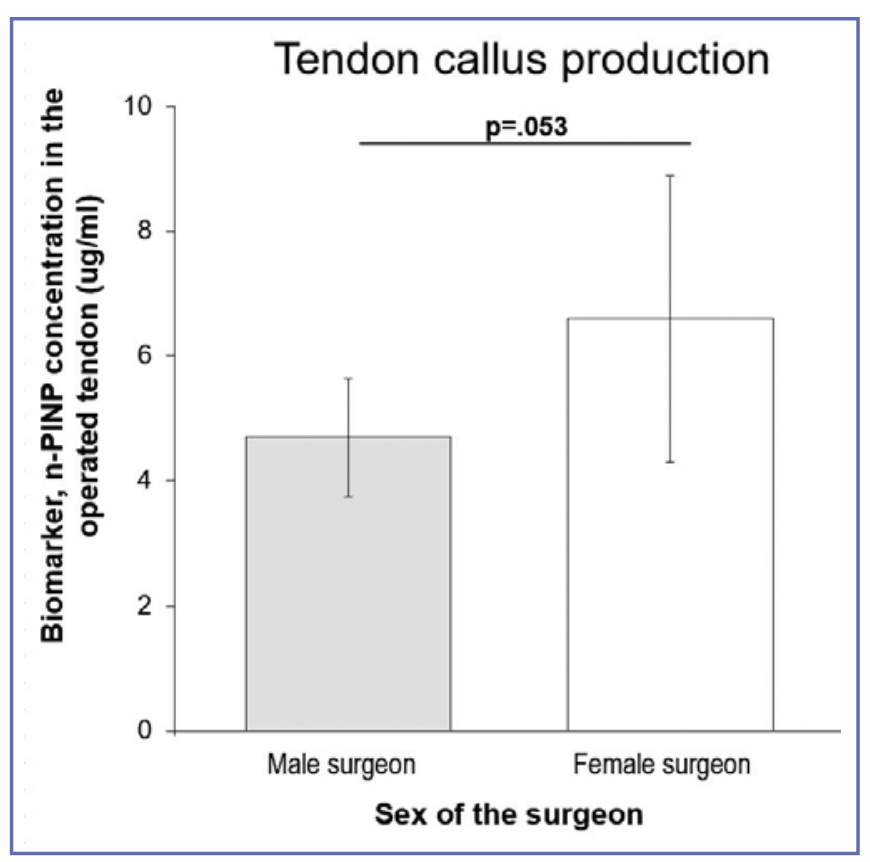

Figure 3. Mean n-PINP concentration in the injured leg for patients operated on by female $(n=19)$ and male surgeons $(n=43)$ at the two-week follow-up microdialysis with $95 \% \mathrm{Cl}$. Regression analyses verified significant differences $(p=0.034)$. 62 patients were included in this analysis.

on by the female surgeons demonstrated significantly less limitations in ADL, less stiffness and more strength in their repaired Achilles tendon according to the self-estimated ATRS. These observations at one-year post-surgery hypothetically reflect differences in surgical techniques, but may also be influenced by the care and information given to the patient post-operatively and during the two- and six-weeks follow-up visits.

There is only a limited number of other reports, exclusively register/database studies, investigating differences in outcome among female and male surgeons. One study including 104630 patients and comparing the outcome of 25 different types of surgeries, demonstrated that patients operated on by female surgeons had a small, but statistically significant, decrease in complications and 30-day mortality (22). The study could, however, not find any potential explanation for the differences observed, but the fact that patients operated on by female surgeons exhibited better outcome seems to support the result of our study.

Two other studies seem to contradict the results of the present study by demonstrating that patient outcomes were similar when surgery was performed by female and male surgeon after matching the age and clinical experience of the 
surgeons $(18,19)$. A third study found that "female surgeons in their 50s had the lowest operative mortality", but the study could, however, not find any evidence that mortality differed between female and male surgeons after adjusting for confounding factors. The present study did also investigate the age of the surgeon and corrected for differences in the experience of the surgeon. The male surgeons had significantly higher age than the female surgeons, but the age of the surgeon was not significantly correlated to any of the patient outcome variables or adverse events.

This study was unable to find any significant differences in the incidence of adverse events (peri-operative surgical complications, DVTs, wound infections and re-ruptures) between patients operated on by male versus female surgeons. This indicates that the improved results seen at two weeks in healing biology and at one year in subjective outcome of patients operated on by female surgeons are not explained by a difference in early complications between the two different surgeon groups.

The second main finding of this study pertained to that patients operated on by female surgeons exhibited significantly higher levels at two weeks post-operatively of n-PINP (procollagen type I) in the repairing tendon.

A few previous studies have demonstrated that higher levels of the tendon healing marker n-PINP assessed at the two-week follow-up are correlated to improved patient outcomes at one year $(1,8,12)$. The in this study observed increased levels of n-PINP, which is one of the major building stones in tendon healing, suggests a plausible explanation for the better patient-reported outcome in patients operated on by female surgeons. The underlying cause of the increased n-PINP is, however, unknown. Hypothetical factors that may alter n-PINP expression are differences in surgical techniques and dissimilarities in post-operative care and information leading to eg. discrepancies in mobilization regime.

The present study is, according to the best of our knowledge, the first non-register study in the field of surgery with data solely collected from RCTs and with all patients undergoing the same surgical procedure, that investigates if the sex of the surgeon affects patient-reported outcomes. Earlier studies, which found that patients operated on by female surgeons exhibited better outcome, could not find a potential explanation for the difference (22). The present study did observe increased levels of n-PINP, which suggests an explanation for the better patient-reported outcome in patients operated on by the female surgeons. However, this conclusion warrants further studies to replicate the findings and examine their underlying factors.

Other factors in patient care, however, have been found to differ between female and male doctors, such as female physicians using more patient-centered communication $(2$,
20). The biological factors pointing to the observed differences related to tendon healing then again strengthen the assumption that there are dissimilarities in care related to the sex of the surgeon that might affect the final patient outcomes. Our results, however, do not support that surgeons should be selected based on their sex, rather they support sex equality also in the surgical field (9). The findings of this study also support the need for further studies on surgical outcomes related to surgical techniques, surgeon characteristics and surgeon communication, which can be used to improve patient outcomes.

The study did have some limitations and although we controlled for several possible confounding factors, the study was a retrospective cohort-study with all its potential bias. The two cohorts included exhibited small differences in the post-operative treatment, however this was controlled for in the statistical analyses and had no significant effects on the outcome. The rehabilitation of the patients was closely monitored during the first six weeks following surgery, where after patients were given clear instructions on how to continue their rehabilitation. Beyond the six-week timepoint, however, monitoring of individual differences in rehabilitation was not performed. In this study female surgeons performed more surgeries per surgeon (in average 3.4 surgeries per female surgeon and 2.5 surgeries per male surgeon). Thus ATR-surgery volume is a factor that has to be studied further.

An earlier study suggested that a potential bias may be present if patients are given the option to select their surgeon (14). Therefore, a strength of this study was that the patients did not have the option to choose their surgeon, as they were operated on by the surgeon available at the day of surgery. Other potential confounders affecting the results may be patient expectation on the surgeon, how the patients tolerate and judge their symptoms and the surgeon experience of Achilles tendon surgery before this study, which all were factors we could not control for. Therefore, to corroborate our results, prospective randomized controlled trials comparing the surgeons are needed.

\section{CONCLUSIONS}

The present study shows no significant differences in adverse events in ATR patients related to the sex of the surgeon, but it does suggest that patients operated on by the female surgeons exhibited superior patient-reported outcomes (a mean of 7 points higher total ATRS) one year after ATR-surgery. We also found that patients operated on by the female surgeons exhibited higher levels of important healing biomarkers. There is no single evident explanation for these observations, but plausible factors additional to surgi- 
cal techniques include the care and information given to the patient post-operatively. These findings do not warrant policy changes, but rather further examinations on the surgeon factors underlying ATR healing and patient outcome.

\section{REFERENCES}

1. Alim MA, Svedman S, Edman G, Ackermann PW. Procollagen markers in microdialysate can predict patient outcome after Achilles tendon rupture. BMJ Open Sport Exerc Med 2016:2:1-7.

2. Bertakis KD, Helms LJ, Callahan EJ, Azari R, Robbins JA. The influence of gender on physician practice style. Med Care 1995;359:407-16.

3. Berthold HK, Gouni-Berthold I, Bestehorn KP, Böhm M, Krone W. Physician gender is associated with the quality of type 2 diabetes care. J Intern Med 2008;359:340-50.

4. Chun DS, Cook RW, Weiner JA, Schallmo MS, Barth JA, Singh SK, et al. Can Surgeon Demographic Factors Predict Postoperative Complication Rates After Elective Spinal Fusion? Clin Spine Surg 2018;31:2:93-97.

5. Domeij-Arverud E, Labruto F, Latifi A, Nilsson G, Edman G, Ackermann PW. Intermittent pneumatic compression reduces the risk of deep vein thrombosis during post-operative lower limb immobilisation: a prospective randomised trial of acute ruptures of the Achilles tendon. Bone Joint J 2015;97-B(5):675680.

6. Ganestam A, Barfod K, Klit J, Troelsen A. Validity and reliability of the Achilles tendon total rupture score. J Foot Ankle Surg 2013;52(6):736-9.

7. García-Granero E, Navarro F, Cerdán Santacruz C, García-Granero A, Marinello F, et al. Individual surgeon is an independent risk factor for leak after double-stapled colorectal anastomosis: An institutional analysis of 800 patients. Surgery 2017;162:1006-16.

8. Greve K, Domeij-Arverud E, Labruto F, Edman G, Bring D, Nilsson $G$, et al. Metabolic activity in early tendon repair can be enhanced by intermittent pneumatic compression. Scand J Med Sci Sports 2012;22(4):e55-63

9. Jagsi R, Griffith KA, DeCastro RA, Ubel P. Sex, Role Models, and Specialty Choices Among Graduates of US Medical Schools in 2006-2008. Journal of the American College of Surgeons 2014;218(3):345 - 352 .

10. Kjaer M, Langberg H, Bojsen-Møller J, Koskinen SO, Mackey A, Heinemeier K, et al. Novel methods for tendon investigations. Disabil Rebabil 2008;30(20-22):1514-22.

11. Langberg H, Olesen JL, Bülow J, Kjaer M. Intra- and peri-tendinous microdialysis determination of glucose and lactate in pigs. Acta Pbysiol Scand 2002;174(4):377-80.

\section{CONFLICT OF INTERESTS}

The authors declare that they have no conflicts of interest.

12. Langberg H, Skovgaard D, Karamouzis M, Bülow J, Kjaer M. Metabolism and inflammatory mediators in the peritendinous space measured by microdialysis during intermittent isometric exercise in humans. J Physiol 1995;515 (Pt 3):919-27.

13. Lapidus L, de Bri E, Ponzer S, Elvin A, Noren A, Rosfors S. High sensitivity with color duplex sonography in thrombosis screening after ankle fracture surgery. J Thromb Haemost. 2006;4(4):807-12.

14. Nilsson-Helander K, Thomeé R, Silbernagel KG, Grävare-Silbernagel K, Thomeé P, Faxén E, et al. The Achilles tendon Total Rupture Score (ATRS): development and validation. AJSM 2007;35(3):421-6.

15. Oliva F, Rugiero C, Giai Via A, Baldassarri M, Bernardi G, Biz C, et al. I.S.Mu.L.T. Achilles tendon ruptures guidelines. MLTJ 2018;8(3):310-63.

16. Read TE, Myerson RJ, Fleshman JW, Fry RD, Birnbaum EH, Walz BJ. Surgeon specialty is associated with outcome in rectal cancer treatment. Dis Colon Rectum 2002;45(7):90414.

17. Roter DL, Hall JA, Aoki Y. Physician gender effects in medical communication: a meta-analytic review. JAMA 2002;359:756-64.

18. Sharoky CE, Sellers MM, Keele LJ, Wirtalla CJ, Karakousis GC, Morris JB, et al. Does Surgeon Sex Matter?: Practice Patterns and Outcomes of Female and Male Surgeons. Ann Surg 2018;267(6):1069-76.

19. Tsugawa Y, Jena AB, Orav EJ, Blumenthal DM, Tsai TC, Mehtsun WT, et al. Age and sex of surgeons and mortality of older surgical patients: observational study. BMJ 2018;25;361:k1343.

20. Tsugawa Y, Jena AB, Figueroa JF, Orav EJ, Blumenthal DM, Jha AK. Comparison of hospital mortality and readmission rates for Medicare patients treated by male vs female physicians. JAMA Intern Med 2017;359:206-13.

21. Valkering KP, Aufwerber S, Ranuccio F, Lunini E, Edman G, Ackermann PW. Functional weight-bearing mobilization after Achilles tendon rupture enhances early healing response: a single-blinded randomized controlled trial. KSSTA 2017;25:1807-16.

22. Wallis CJ, Ravi B, Coburn N, Nam RK, Detsky AS, Satkunasivam R. Comparison of postoperative outcomes among patients treated by male and female surgeons. BMI 2017;10;359:j4366. 